\title{
Puncture point-traction method: A novel method applied for right internal jugular vein catheterization
}

\author{
TIANLIANG WU and HONGCHENG ZANG \\ Department of Anesthesia, The First People's Hospital of Fuyang District, Hangzhou, Zhejiang 311400, P.R. China
}

Received February 2, 2015; Accepted March 14, 2016

DOI: $10.3892 /$ etm.2016.3258

\begin{abstract}
The ultrasound probe and advancement of the needle during real-time ultrasound-assisted guidance of catheterization of the right internal jugular vein (RIJV) tend to collapse the vein, which reduces the success rate of the procedure. We have developed a novel puncture point-traction method (PPTM) to facilitate RIJV cannulation. The present study examined whether this method facilitated the performance of RIJV catheterization in anesthetized patients. In this study, 120 patients were randomly assigned to a group in which PPTM was performed (PPTM group, $n=60$ ) or a group in which it was not performed (non-PPTM group, $\mathrm{n}=60$ ). One patient was excluded because of internal carotid artery puncture and 119 patients remained for analysis. The cross-sectional area (CSA), anteroposterior diameter (AD) and transverse diameter (TD) of the RIJV at the cricoid cartilage level following the induction of anesthesia and during catheterization were measured, and the number with obvious loss of resistance (NOLR), the number with easy aspiration of blood into syringe (NEABS) during advancement of the needle, and the number of first-pass punctures (NFPP) during catheterization were determined. In the non-PPTM group, the CSA was smaller during catheterization compared with that following the induction of anesthesia $(\mathrm{P}<0.01)$. In the PPTM group compared with the non-PPTM group during catheterization, the CSA was larger $(\mathrm{P}<0.01)$ and the $\mathrm{AD}(\mathrm{P}<0.01)$ and TD $(\mathrm{P}<0.05)$ were wider; NOLR $(\mathrm{P}<0.01)$, NEABS $(\mathrm{P}<0.01)$ and NFPP $(\mathrm{P}<0.01)$ increased significantly. The findings from this study confirmed that the PPTM facilitated catheterization of the RIJV and improved the success rate of RIJV catheterization in anesthetized patients in the supine position.
\end{abstract}

Correspondence to: Dr Tianliang Wu, Department of Anesthesia, The First People's Hospital of Fuyang District, 2-4 Guihua Road, Fuyang, Hangzhou, Zhejiang 311400, P.R. China

E-mail: liangtianwuwu@163.com

Key words: real-time ultrasound-assisted guidance, right internal jugular vein, puncture point-traction method, suture

\section{Introduction}

Central venous catheterization is a commonly performed procedure used in modern clinical practice (1-3), which is suggested when peripheral veins are inaccessible. Central venous catheterization may be lifesaving, but its performance requires critical operator training and experience. A prior study showed that $>15 \%$ of patients who received central venous catheterization suffered from complications (4). Therefore, methods promotion for reducing the frequency of complications are required. Right internal jugular vein (RIJV) catheterization with ultrasound-assisted guidance has been proposed as a simple and reliable procedure for multimodality intravenous therapy that meets the requirements of central venous catheterization (4-6). Furthermore, real-time ultrasound-assisted guidance has been suggested to improve the success rate of IJV (7). However, the ultrasonic probe and advancement of the puncture needle can cause the vein to collapse, which reduces the success rate of the procedure (8). It has been suggested that the catheter could be modified to minimize the risk of displacement or migration (9). Morita et al developed a novel 'skin traction method' which increased the compressive force required to collapse the IJV, and consequently facilitated catheterization of the IJV (10). In addition, a study by Lim et al in 2012 indicated that the bevel-down approach for needle puncture avoided posterior venous wall damage during IJV catheterization (11). However, as an alternative to this, the present study describes a novel puncture point-traction method (PPTM) that has been developed to facilitate RIJV cannulation, in which it is attempted to retain the puncture point of the skin directly above the RIJV in its original position by the traction of surgical suture. The aim of this study was to assess the effect of the PPTM on RIJV catheterization in anesthetized patients in the supine position and to consequently verify the application this novel PPTM.

\section{Materials and methods}

Ethical approval. The study was approved by the Ethics Committee of Fuyang People's Hospital (Fuyang, China), and informed consent was confirmed by all patients. There were 120 patients who were about to have surgery with RIJV catheterization and suture fixation. The patients were divided into physical status grades I-III according to the American Society of Anesthesiologists (ASA) classification system (12). Their 
average age was $65.2 \pm 11.4$ years (range, $19-73$ years), with an average body weight of $61.9 \pm 7.1 \mathrm{~kg}$ (range, $48-82 \mathrm{~kg}$ ) and average height of $168.4 \pm 8.2 \mathrm{~cm}$ (range, $160-183 \mathrm{~cm}$ ) (Table I). All patients were randomly divided into a PPTM group $(n=60$, treated with the PPTM method) and a non-PPTM group ( $\mathrm{n}=60$, treated without the PPTM method). Patients were not included in this study according to the following exclusion criteria: External neck injury, previous history of RIJV catheterization, severe cardiovascular disease, injection-site infection, hematological disease, thrombogenesis history, obesity and short-term venous catheterization.

Surgical procedure. Heart rate (HR), oxygen saturation and mean arterial pressure (MAP) were measured continuously. In cases where the MAP decreased to $<60 \mathrm{mmHg}$ and the HR decreased to $<60$ beats per minute during venipuncture, which was identified as hypotension and bradycardia, all patients were placed in the supine position without using pillows, with the head turned $30^{\circ}$ to the left following the induction of anesthesia. Remedication with $0.1 \mathrm{mg} / \mathrm{kg}$ intravenous midazolam was given $10 \mathrm{~min}$ before induction. Induction was performed using $2 \mu \mathrm{g} / \mathrm{kg}$ fentanyl followed by $2.5 \mathrm{mg} / \mathrm{kg}$ propofol. Tracheal intubation was facilitated using rocuronium with a dose of $1.2 \mathrm{mg} / \mathrm{kg}$. An ultrasound probe (L25x, 13-6 MHz; SonoSite, Inc. Bothell, WA, USA) was used during catheterization, which was applied perpendicular to the skin without any pressure. The cross-sectional area (CSA), anteroposterior diameter (AD) and transverse diameter (TD) of the RIJV at the cricoid cartilage level were measured with three replicates using electronic calipers on the ultrasound image in all patients following the induction of anesthesia and during catheterization (when aspiration of blood into the syringe occurred during advancement of the needle).

In the PPTM group, the skin directly above the RIJV at the level of the cricoid cartilage was identified by ultrasound following disinfection of the right neck skin. A surgical suture (Péters Surgical, Bobigny, France) was sutured at this position, without tying a knot, using a skin-suturing needle. The suture was intradermal for $1 \mathrm{~cm}$ with $20-\mathrm{cm}$ lengths of suture outside the skin at each side. Cannulation was put into place under real-time ultrasound visualization in the transverse plane. During catheterization, the two ends of the suture were wound around the left hand thumb of operator and were held closely to the middle of the ultrasonic probe, which was directed at the side of the patient's head (Fig. 1A). A puncture needle (16 G, $20 \mathrm{~cm}$; Lepu Medical Technology Co., Ltd., Beijing, China) was advanced slowly and carefully under continuous negative pressure at the position of the suture in the skin. The angle of the needle was $35-45^{\circ}$ to the skin. During advancement of the needle, the position of the ultrasonic probe remained unchanged, and the suture was straightened with the advancement of the needle. When the ultrasound probe did not touch the patient's skin, they were brought into contact by pulling the suture using the left thumb. When blood entered the needle, the position of the needle was maintained using the left hand and a guidewire was inserted. Consequently, RIJV catheterization was carried out, and a suture was applied to fix the catheter (Fig. 1B). In the non-PPTM group, only when the RIJV catheterization at the level of the cricoid cartilage was completed was the
Table I. General characteristics of all patients.

\begin{tabular}{lcc}
\hline Variable & $\begin{array}{c}\text { Non-PPTM group } \\
(\mathrm{n}=59)\end{array}$ & $\begin{array}{c}\text { PPTM group } \\
(\mathrm{n}=60)\end{array}$ \\
\hline Age, years & $65.6 \pm 12.4$ & $64.8 \pm 10.8$ \\
Height, $\mathrm{cm}$ & $168.5 \pm 8.3$ & $168.2 \pm 7.8$ \\
Weight, $\mathrm{kg}$ & $61.6 \pm 7.2$ & $62.4 \pm 7.4$ \\
Gender, male/female & $37 / 22$ & $34 / 26$ \\
ASA class I/II/III & $24 / 31 / 4$ & $27 / 26 / 7$ \\
\hline
\end{tabular}

Data are expressed as mean \pm standard deviation or number of patients. PPTM, puncture point-traction method; ASA, American Society of Anesthesiologists.

position of the suture selected and the suture applied to fix the catheter. The remaining procedures of the surgery in the non-PPTM group were the same as those in the PPTM group. In cases where the right internal carotid artery (ICA) was punctured in either group, RIJV catheterization was abandoned.

Measurements. Age, gender, height, weight and ASA grades of the patients were recorded. The following variables were also recorded: CSA, AD and TD of the RIJV at the cricoid cartilage level following anesthesia induction and during catheterization (when aspiration of blood into syringe occurred during advancement of needle) in the two groups. Measurements were performed three times for each condition. The number with obvious loss of resistance (NOLR), the number with easy aspiration of blood into syringe (NEABS) during advancement of the needle, the depth of needle insertion (DNI), the number of first-pass punctures (NFPP) and total success rate (TSR) were examined. In addition, the HR, minimum value of MAP, hypotension, bradycardia and puncture-related complications such as pneumothorax, hemopneumothorax, local hematoma and ICA puncture during catheterization were recorded.

Statistical analysis. SPSS statistical analysis software, version 13.0 (SPSS, Inc., Chicago, IL, USA) was applied for statistical analysis and data values are presented as mean \pm standard deviation. Statistically significant differences between groups were assessed using one-way analysis of variance followed by the Bonferroni multiple comparison test to evaluate intergroup differences. $\mathrm{P}<0.05$ indicated a statistically significant difference.

\section{Results}

Changes in CSA, AD and TD. The CSA $(\mathrm{P}<0.01), \mathrm{AD}$ $(\mathrm{P}<0.01), \mathrm{TD}(\mathrm{P}<0.05)$ of the RIJV at the cricoid cartilage level in the non-PPTM group were significantly smaller during catheterization compared with those following the induction of anesthesia (Fig. 2 and Table II); however, no significant differences $(\mathrm{P}>0.05)$ in these measurements between these two time points were observed in the PPTM group (Fig. 3 and Table II). The CSA ( $\mathrm{P}<0.01), \mathrm{AD}(\mathrm{P}<0.01)$, 
Table II. Changes in CSA, AD, and TD of the RIJV at the cricoid cartilage level following the induction of anesthesia and during catheterization, and other venipuncture-related data for the two groups.

\begin{tabular}{lcc} 
Outcome & Non-PPTM group & PPTM group \\
\hline CSA, $\mathrm{cm}^{2}$ & & $1.61 \pm 0.31$ \\
After anesthesia induction & $1.63 \pm 0.38$ & $1.72 \pm 0.33^{\mathrm{b}}$ \\
During catheterization & $0.93 \pm 0.21^{\mathrm{a}}$ & \\
AD, cm & & $1.54 \pm 0.67$ \\
After anesthesia induction & $1.55 \pm 0.73$ & $1.48 \pm 0.50^{\mathrm{b}}$ \\
During catheterization & $1.02 \pm 0.16^{\mathrm{a}}$ & \\
TD, cm & & $3.40 \pm 1.31$ \\
After anesthesia induction & $3.39 \pm 1.33$ & $3.38 \pm 0.29^{\mathrm{d}}$ \\
During catheterization & $2.18 \pm 0.28^{\mathrm{c}}$ & $55(91.7)^{\mathrm{b}}$ \\
NOLR, $\mathrm{n}(\%)$ & $4(6.78)$ & $53(88.3)^{\mathrm{b}}$ \\
NEABS during advancement of the needle, $\mathrm{n}(\%)$ & $28(47.5)$ & $56(93.3)^{\mathrm{b}}$ \\
NFPP, $\mathrm{n}(\%)$ & $40(67.8)$ & \\
\hline
\end{tabular}

Data are expressed as mean \pm standard deviation, or number $(\mathrm{n})$ and percentage $(\%)$. ${ }^{\mathrm{a}} \mathrm{P}<0.01 \mathrm{vs}$. after anesthesia induction; ${ }^{\mathrm{P}} \mathrm{P}<0.01 \mathrm{vs}$. the non-PPTM group; ${ }^{c} \mathrm{P}<0.05$ vs. after anesthesia induction; ${ }^{\mathrm{d}} \mathrm{P}<0.05$ vs. the non-PPTM group. CSA, cross-sectional area; $\mathrm{AD}$, anteroposterior diameter; TD, transverse diameter; RIJV, right internal jugular vein; PPTM, puncture point-traction method; NOLR, number with obvious loss of resistance; NEABS, number with easy aspiration of blood into syringe; NFPP, number of first-pass punctures.

A

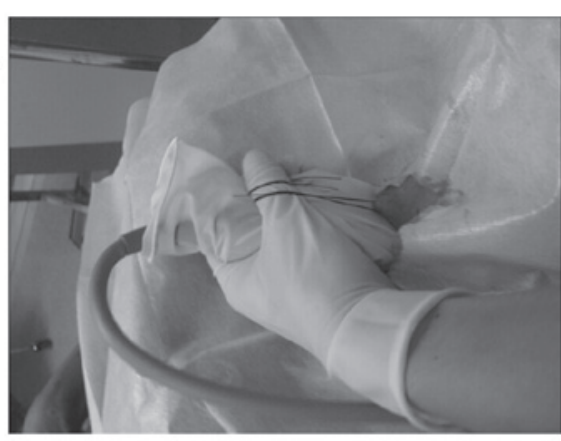

B

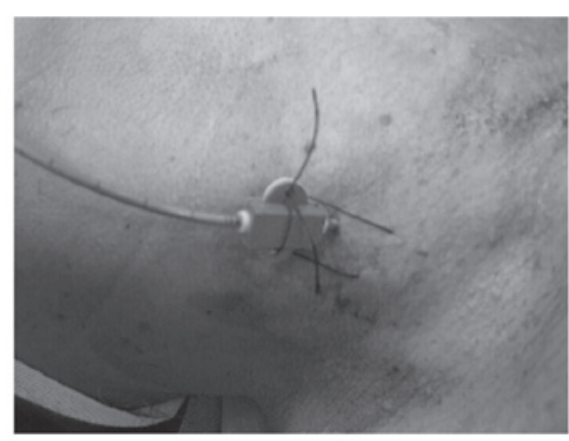

Figure 1. (A) In the PPTM group, the suture was wound around the left thumb for two turns, and was held closely to the middle of the ultrasonic probe, which was pointed at the side of the head during catheterization. (B) In the PPTM group, a suture was applied to fix the RIJV catheter following the successful puncture. PPTM, puncture point-traction method; RIJV, right internal jugular vein.

A

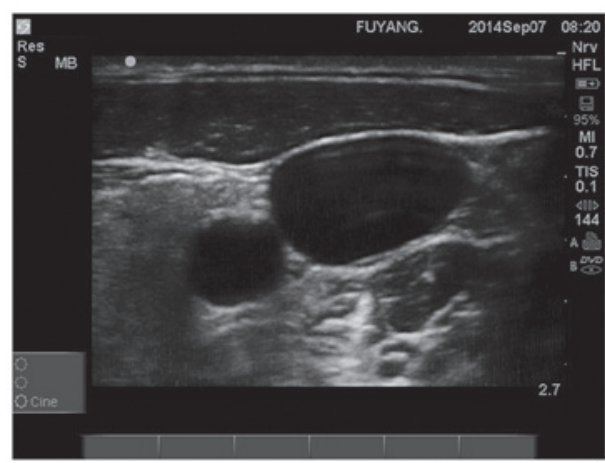

B

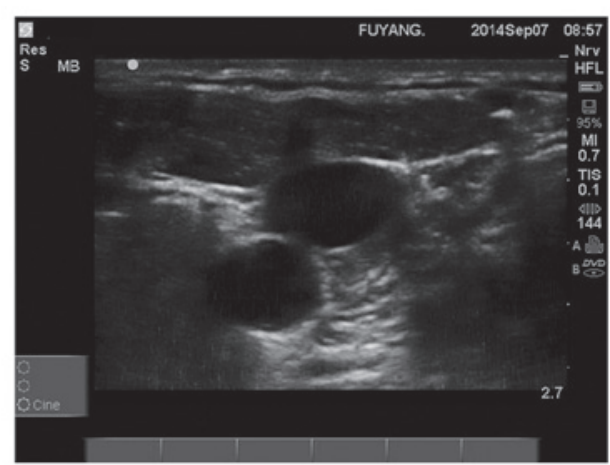

Figure 2. The CSA, AD and TD at the cricoid cartilage level were higher (A) following the induction of anesthesia in the non-PPTM group than (B) during catheterization. CSA, cross sectional area; AD, anteroposterior diameter; TD, transverse diameter; PPTM, puncture point-traction method.

TD $(\mathrm{P}<0.05)$ of the RIJV at the cricoid cartilage level during catheterization were larger in the PPTM group compared with those in the non-PPTM group (Table II).
Changes in NOLR, NEABS and NFPP. During needle insertion and catheterization, the NOLR $(\mathrm{P}<0.01)$, NEABS $(\mathrm{P}<0.01)$ and NFPP $(\mathrm{P}<0.01)$ in the PPTM group were significantly 
A

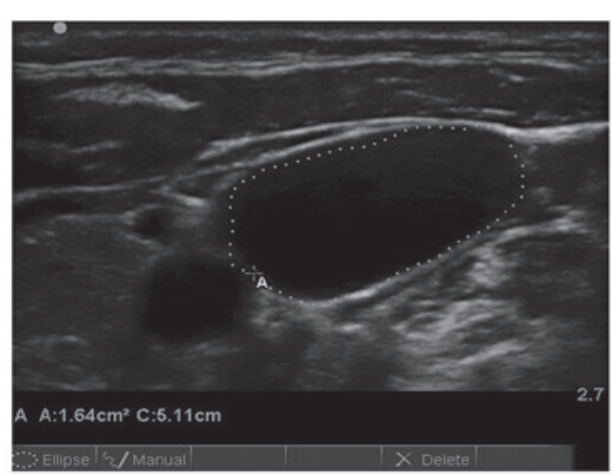

B

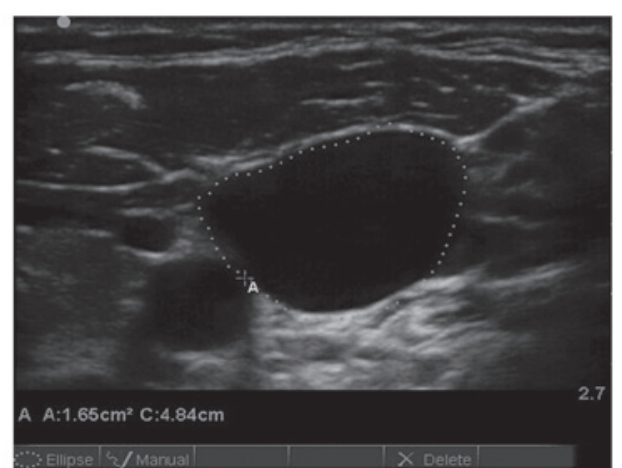

Figure 3. CSA of the RIJV at the cricoid cartilage level (A) following the induction of anesthesia was comparable with that (B) during catheterization when the puncture point-traction method was used. CSA, cross sectional area; RIJV, right internal jugular vein.

increased compared with those in the non-PPTM group (Table II). However, the DNI, catheterization time and TSR were not significantly different during catheterization between the two groups $(\mathrm{P}>0.05$; data not shown).

Complications. There was one case of exclusion from the study because of ICA puncture in the non-PPTM group. Apart from this, there were no other complications in either of the two groups.

\section{Discussion}

Safe and efficient placement of central venous catheters is an essential skill in many fields of clinical medicine, which is mainly conducted by direct visualization methods. Some studies (13-17) have indicated that ultrasound-assisted guidance of internal jugular vein catheterization increases the success rate, and reduces the catheterization time and puncture complications. However, several studies (18-20) have found that perforation of the posterior vessel wall continues to occur, even when venipuncture is guided by ultrasound. Studies $(21,22)$ have found that ultrasound-assisted guidance cannot completely avoid ICA puncture because application of the ultrasound probe and advancement of the needle cause RIJV collapse. Gordon et al (21) have observed that the CSA and the diameter of the vein influence the success rate of central vein catheterization. In anesthetized patients, positive end-expiratory pressure or the Trendelenberg position increase the CSA of the IJV $(23,24)$. It was found in the present study that the RIJV could be maintained in a state of natural fullness by the PPTM, and it prevented the RIJV from collapsing due to stretching of the skin directly above the RIJV, with a success rate of $100 \%$.

It was observed in the present study that the CSA, AD and TD of the RIJV at the cricoid cartilage level were smaller during catheterization than those after anesthesia induction in the non-PPTM group, which may be attributed to the pressure from the ultrasonic probe and the advancement of needle. However, there was no significant change in the CSA, AD and TD of the RIJV at the cricoid cartilage level between after the induction of anesthesia and during catheterization in the PPTM group. A sudden loss of resistance occurred in $91.7 \%$ of the patients during venipuncture in the PPTM group, and it was found that only the anterior vascular wall was perforated in these patients. Additionally, blood was easily aspirated in
$88.3 \%$ of the patients in the PPTM group, which implied that only the anterior vascular wall was perforated, and it was found that the advanced needle did not touch the posterior vascular wall. These findings confirmed that PPTM helped to maintain the natural state of fullness of the RIJV during catheterization by providing a counterforce against the pressure created by the advancement of the needle and the ultrasonic probe, and the PPTM helped the smooth backflow of blood without repeated venipuncture.

During venipuncture, we recommend that the suture is pulled closely to the middle of the ultrasonic probe, which is pointed at the side of the patient's head. The height of the ultrasonic probe was kept unchanged, the needle tip was inserted in a downward direction and it was found that the PPTM reduced the pressure on the RIJV caused by the ultrasonic probe and the advancement of needle, and maintained the skin directly above the RIJV in its original position. If the height of the ultrasonic probe was reduced with advancement of the needle, the CSA, AD and TD of the RIJV at the cricoid cartilage level would be influenced by the pressure of the ultrasonic probe. Therefore, the height of the ultrasonic probe was kept unchanged. Denys et al (6) concluded that the success rate of FPP was $78 \%$ using a $16-\mathrm{G}$ puncture needle. In the present study, a rather higher success rate of FPP (93.3\%) was attained by the PPTM.

Verghese et al (25) suggested that the IJV might be collapsed by advancement of the needle, which would increase the possibility of accidental ICA puncture. In the present study, it was found that the CSA, AD and TD of the RIJV at the cricoid cartilage level were relatively smaller in the non-PPTM group than those in the PPTM group during venipuncture, so the possibility of accidental right ICA puncture was higher in the non-PPTM group than in the PPTM group. One case was excluded from the present study because of ICA puncture in the non-PPTM group, although there was no significant difference between the two groups with regard to success rate.

In our preliminary experiments, it was found that suture slippage was more likely when pulling the suture if the ends of the suture were wound around the right thumb pulp for less than two turns (data not shown). Therefore, it was decided that at least two turns of suture winding were necessary. A decision was also made to pull the suture 1-2 mm above the neck skin, as it was found that the ultrasonic probe would not touch the skin when the puncture needle was inserted without 
pulling the suture. If excessive force was applied on the suture, the CSA of the RIJV at the cricoid cartilage level would be shown only partly in the ultrasound imaging, and the AD at the cricoid cartilage level would also be increased. Ultrasound imaging of the whole RIJV could not be obtained completely when the length of suture beneath the skin was too short or was not in parallel with the cricoid cartilage; therefore, it was decided to keep the suture $>1 \mathrm{~cm}$ beneath the skin in parallel with the cricoid cartilage during venipuncture.

In conclusion, the findings from this study confirmed that the PPTM method facilitated the catheterization of RIJV and improved the success rate of RIJV catheterization in anesthetized patients in the supine position.

\section{Acknowledgements}

The authors would like to thank Fuyang People's Hospital for financial support.

\section{References}

1. de Jonge RC, Polderman KH and Gemke RJ: Central venous catheter use in the pediatric patient: Mechanical and infectious complications. Pediatr Crit Care Med 6: 329-339, 2005.

2. Sanford TJ Jr: Internal jugular vein cannulation versus subclavian vein cannulation. An anesthesiologist's view: The right internal jugular vein. J Clin Monit 1: 58-61, 1985.

3. Defalque RJ: Percutaneous catheterization of the internal jugular vein. Anesth Analg 53: 116-121, 1974.

4. McGee DC and Gould MK: Preventing complications of central venous catheterization. N Engl J Med 348: 1123-1133, 2003.

5. Chuan WX, Wei W and Yu L: A randomized-controlled study of ultrasound prelocation vs anatomical landmark-guided cannulation of the internal jugular vein in infants and children. Pediatr Anesth 15: 733-738, 2005.

6. Denys BG, Uretsky BF and Reddy PS: Ultrasound-assisted cannulation of the internal jugular vein. A prospective comparison to the external landmark-guided technique. Circulation 87: $1557-1562,1993$.

7. Takeji S, Hiroshi S, Azumi K, Mamoru N, Masashi M,Takamitsu T, Katsunori S, Masao S, Tsuyoshi U, Hideki K and Hideharu H: Ultrasound analysis of the relationship between right internal jugular vein and common carotid artery in the left head-rotation and head-flexion position. Heart Vessels 28: 620-625, 2013.

8. Sasano H, Morita M, Azami T, Ito S, Sasano N, Kato R, Hirate H, Ito H, Takeuchi A and Sobue K: Skin-traction method prevents the collapse of the internal jugular vein caused by an ultrasound probe in real-time ultrasound-assisted guidance. J Anesth 23: 41-45, 2009

9. Redo SF: Modified catheter for prolonged central venous use. J Pediatr Surg 30: 1544-1545, 1995.

10. Morita M, Sasano H, Azami T, Sasano N, Fujita Y, Ito S and Sobue K: A novel skin-traction method is effective for real-time ultrasound-guided internal jugular vein catheterization in infants and neonates weighing less than 5 kilograms. Anesth Analg 109: 754-759, 2009.
11. Lim T, Ryu HG, Jung CW, Jeon Y and Bahk JH: Effect of the bevel direction of puncture needle on success rate and complications during internal jugular vein catheterization. Crit Care Med 40: 491-494, 2012.

12. Wolters U, Wolf T, Stützer H and Schröder T: ASA classification and perioperative variables as predictors of postoperative outcome. Br J Anaesth 77: 217-222, 1996.

13. Legler D and Nugent M: Doppler localization of the internal jugular vein facilitates central venous cannulation. Anesthesiology 60: 481-482, 1984.

14. Randolph AG, Cook DJ, Gonzales CA and Pribble CG: Ultrasound guidance for placement of central venous catheters: A meta-analysis of the literature. Crit Care Med 24: 2053-2058, 1996.

15. Hind D, Calvert N, McWilliams R, Davidson A, Paisley S, Beverley $\mathrm{C}$ and Thomas S: Ultrasonic locating devices for central venous cannulation: meta-analysis. British Medical Journal 327: 361-364, 2003.

16. Mallory DL, McGee WT, Shawker TH, Brenner M, Bailey KR, Evans RG, Parker MM, Farmer JC and Parillo JE: Ultrasound guidance improves the success rate of internal jugular vein cannulation. A prospective, randomized trial. Chest 98: 157-160, 1990.

17. Karakitsos D, Labropoulos N, De Groot E, Patrianakos AP, Kouraklis G, Poularas J, Samonis G, Tsoutsos DA, Konstadoulakis MM and Karabinis A: Real-time ultrasound-guided catheterisation of the internal jugular vein: A prospective comparison with the landmark technique in critical care patients. Crit Care 10: R162, 2006.

18. Blaivas M and Adhikari S: An unseen danger: Frequency of posterior vessel wall penetration by needles during attempts to place internal jugular vein central catheters using ultrasound guidance. Crit Care Med 37: 2345-2349, 2009.

19. Ganesh A and Jobes DR: Ultrasound-guided catheterization of the internal jugular vein. Anesthesiology 108: 1155-1156, 2008.

20. Mallory DL, Shawker T, Evans RG, McGee WT, Brenner M, Parker M, Morrison G, Mohler P, Veremakis C and Parrillo JE: Effects of clinical maneuvers on sonographically determined internal jugular vein size during venous cannulation. Crit Care Med 18: 1269-1273, 1990.

21. Gordon AC, Saliken JC, Johns D, Owen R and Gray RR: US-guided puncture of the internal jugular vein: Complications and anatomic considerations. J Vasc Interv Radiol 9: 333-338, 1998.

22. Leung J, Duffy M and Finckh A: Real-time ultrasonographically-guided internal jugular vein catheterization in the emergency department increases success rates and reduces complications: A randomized, prospective study. Ann Emerg Med 48: 540-547, 2006.

23. Lobato EB, Florete OG Jr, Paige GB and Morey TE: Cross-sectional area and intravascular pressure of the right internal jugular vein during anesthesia: Effects of Trendelenburg position, positive intrathoracic pressure and hepatic compression. J Clin Anesth 10: 1-5, 1998 .

24. Troianos CA, Jobes DR and Ellison N: Ultrasound-guided cannulation of the internal jugular vein. A prospective, randomized study. Anesth Analg 72: 823-826, 1991.

25. Verghese ST, McGill WA, Patel RI, Sell JE, Midgley FM and Ruttimann UE: Ultrasound-guided internal jugular venous cannulation in infants: A prospective comparison with the traditional palpation method. Anesthesiology 91: 71-77, 1999. 